DEVELOPMENT

\section{It's all in the timing}

The signalling factor Sonic hedgehog $(\underline{\mathrm{SHH}})$ has been shown to specify the identity of motor and interneurons in the developing spinal cord in a concentration-dependent manner. Bai and colleagues now report that SHH does not act alone: WNT signalling lends a helping hand in a time-dependent fashion.

$\mathrm{SHH}$ activates the transcription of different combinations of homeobox and basic helix-loop-helix

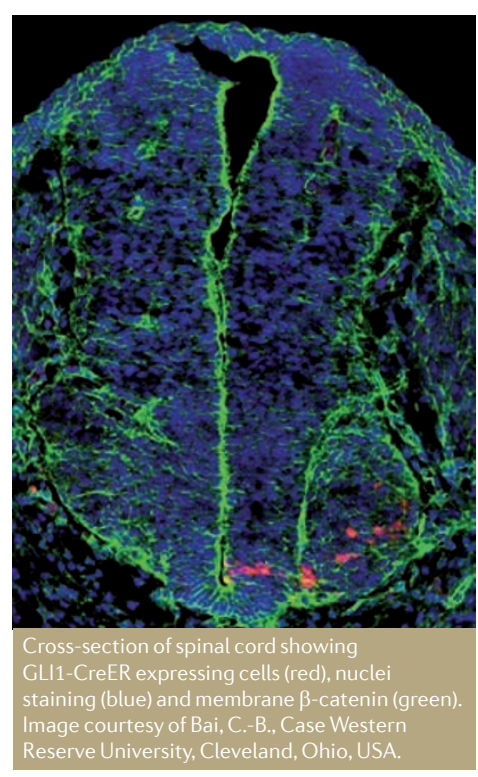

transcription factors that define the fate of neural progenitors in the ventral spinal cord. However, it was suspected that other signals could be involved in this process as several of these cell types are generated even when the cells cannot respond to SHH signalling.

Canonical WNT signalling results in the stabilization of $\beta$-catenin. Therefore, to examine the effects of activating this pathway, the authors induced the expression of stabilized $\beta$-catenin in small patches of neuronal progenitors in mouse spinal cord. They found that stabilized $\beta$-catenin induced the expression of the dorsal cell markers PAX7, MSX1, MSX2 and MASH1 (also known as ASCL1) and inhibited the expression of the ventral cell markers NKX2.2, OLIG2 and NKX6.1 in the ventral spinal cord. In agreement with these findings, they observed an expansion of ventral cell types in mutant mice in which the expression of $\beta$-catenin was drastically reduced in the developing ventral spinal cord.

Interestingly, depending on the developmental stage at which $\beta$-catenin was stabilized, different subsets of genes were expressed, suggesting that WNT signalling can specify different cell fates in a time-dependent manner - effects of signal strength and duration, although possible, are likely to be negligible given that expression of stabilized $\beta$-catenin maximizes WNT signal strength and that extending the duration of active WNT signalling did not alter the expression of dorsal markers.

To gain some insight into the mechanisms that underlie these effects, the authors examined whether $\beta$-catenin stabilization affected the expression of the mediators of SHH signalling, GLI2 and GLI3. They found an upregulation of GLI3 in cells expressing stabilized $\beta$-catenin. However, when GLI3 was removed, the WNT-mediated repression of ventral cell fate was not completely lost. Similarly, removal of GLI2 did not affect the WNTinduced cell fate switching, leading the authors to conclude that the effect of WNT signalling on cell fate specification is partially dependent on GLI3, and that GLI2 is not involved. These findings ascribe yet another function to the multitasking WNT pathway in neural development and highlight a direct interaction with SHH signalling.

Monica Hoyos Flight

ORIGINAL RESEARCH PAPER Yu, W.,

McDonnell, K., Taketo, M.-M. \& Bai, C.-B. Wnt signaling determines ventral spinal cord cell fates in a time-dependent manner. Development 135, 3687-3696 (2008) 